\title{
Research on Properties of X-Ray Detection Film Based on Thallium Doped Cesium lodide
}

\author{
Yang Yanbei ${ }^{1, a}$, Tian Chunhui ${ }^{2, b}$, Liu Shuang ${ }^{3, c^{*}}$ \\ ${ }^{1,2,3}$ School of Optoelectronic Science and Engineering, University of Electronic Science and Technology of China
}

\begin{abstract}
As X-ray detection imaging has a wide range of applications in medicine, industry, public safety, etc., it is of great significance to study its imaging mechanism and improve its imaging performance. Based on the process of X-ray luminescence in the scintillator material, this paper established a simulation model using a microcrystalline column structure to investigate the relationship between the thickness of the detection film and the light conversion efficiency. With the help of the simulation tool MATLAB, the Monte Carlo method was used to simulate the light conversion process of X-ray in the film, and the results were obtained as follows. Under the condition of other parameters unchanged, the luminous efficiency reached the peak value with the increase of the film thickness, and then gradually decreased with the increase of film thickness. The reason why the conversion efficiency in the early stage increases with the increase of the film thickness is that the film is in a saturated state, and increasing the thickness can cause more X-ray particles to be converted. As the film thickness increases, more fluorescent photons are absorbed as they propagate in the film, resulting in a gradual decrease in conversion efficiency. Therefore, an appropriate film thickness can be selected based on the simulation results to obtain the ideal light conversion efficiency.
\end{abstract}

\section{Introduction}

X-rays have a very strong penetrating ability. When Xrays penetrate an object, each part of the object will absorb $\mathrm{X}$-rays to varying degrees, which can also be regarded as modulating X-rays. The modulated rays carry structural information inside the object, and then convert the information carried by the X-rays into image information to realize X-rays imaging. Key researches on X-ray imaging have been carried out at home and abroad. The Shanghai Institute of Ceramics used non-vacuum technology to successfully prepare thallium-doped cesium iodide crystals in 2004, which overcomes the defects of the traditional international vacuum growth method. In 2009, the Department of Nuclear and Quantum Engineering of Korea Advanced Institute of Technology developed and tested the pixelated CsI:Tl scintillator film for X-ray image sensors. In 2015, Siegen University in Germany reported a high-energy particle detector composed of cesium iodide and silicon. By combining a low-noise fully depleted CCD detector with a CsI:Tl scintillation screen, a high quantum efficiency (QE) energy dispersion area detector can be realized in the range of less than $1 \mathrm{keV}$ to more than $100 \mathrm{keV}$. With the continuous development of technology, X-ray detection technology has been widely used in industry, medicine and other fields. At present, scintillator detector has attracted

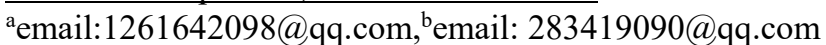

*corresponding author:cemail: shuangliu@uestc.edu.cn much attention for its low cost and excellent performance, and it is a key part of the X-ray detection imaging process. Research on how to improve the light conversion performance of the scintillator is of great significance for improving the imaging quality. This paper established a theoretical model and used Monte Carlo simulation to study the relationship between the thickness of the scintillator material and the light conversion efficiency, which can not only improve the clarity of imaging, but also optimize the structure of imaging equipment on this basis, which has positive significance for the development of imaging technology.

\section{Research and simulation methods}

\subsection{Principle of imaging}

According to quantum theory, the reason why X-rays can excite photons after entering the scintillator is that the inner electrons of the atoms in the scintillator are excited by the X-rays and jump to high-energy electron orbits to generate a hole. The outer electrons of the scintillator atoms fill the holes generated by the excited electrons, release energy, and produce fluorescence. During this whole process, Compton effect and photoelectric effect will occur, as shown in Figure 1. 

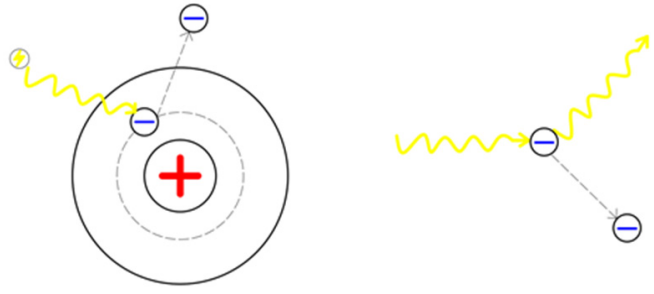

Figure 1 Photoelectric effect and Compton effect

The principle of X-ray detection and imaging is shown in Figure 2. Since the X-ray entering the scintillator is modulated by the object, its intensity is the information of the object's structure, and the number of photons excited by X-rays of different intensities is different, so the light and shade of the image can reflect the information of the object structure. In this way, how to ensure the directional transmission of the excited photons of the scintillator material and the sensitivity of the scintillator material to $\mathrm{X}$-rays is the focus of the next research direction. Since the crystalline pillar formed during the growth of the film material has a function similar to that of an optical fiber, the natural microcrystalline pillar structure can effectively solve the directional transmission of photons, and increasing the light conversion efficiency of the scintillator material can ensure its sensitivity to X-rays.

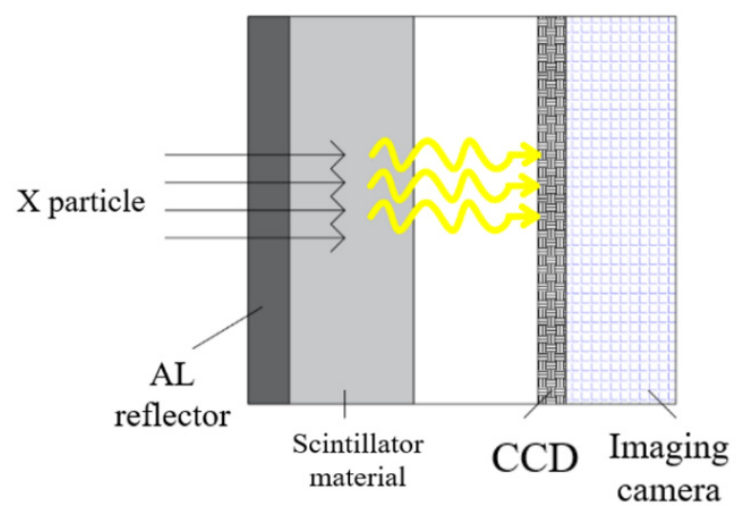

Figure 2 Principle diagram of imaging principle

\subsection{Modeling and simulation}

Since the area of each unit of the detection film is small, the incident X-ray can be approximated as a continuous ideal layer structure with vertical incidence. Assuming that the emitted photons of the X-ray point source are only monochromatic photons with energy $E_{0}$, when the X-ray incident depth is $z$, the attenuation energy can be expressed as:

$$
E_{z}=E_{0} e^{-\mu \rho z}
$$

In formula (1), $\mu$ is the absorption coefficient of CsI:Tl crystal for X-ray particles, in $\mathrm{cm}^{2} / g$; $\rho$ is the density of CsI:Tl crystal, in $\mathrm{g} / \mathrm{cm}^{3}$. Differentiate formula (1) to $\mathrm{z}$ to obtain the X-ray energy absorbed by the CsI:Tl crystal per unit length:

$$
d E_{z}=-\mu \rho E_{0} e^{-\mu \rho z} d z
$$

If the crystalline pillar is divided into a layer thickness of 1 um from top to bottom, the energy absorbed by the $i$ th crystal layer is:

$$
E_{i}=-\mu \rho E_{0} e^{-\mu \rho i}
$$

Obtained by the empirical formula, every $20 \mathrm{eV}$ of energy is absorbed, and a visible photon is produced. And the direction of the photons generated by each layer of film satisfies free excitation (direction angle obeys random distribution). Based on this, we can get the number and direction of photons generated in each film.

Supposing the film thickness is $L$, the ratio of the ray energy absorbed by the scintillator film due to the photoelectric effect to the incident ray energy in the thickness $\mathrm{z} \sim \mathrm{z}+\mathrm{dz}$ at the point $Q(x, y, z)$ is:

$$
f_{z}(z) d z=\frac{\frac{\mu_{p e}}{\mu}\left|d E_{z}\right|}{E_{0}}=\mu_{p e} e^{-\mu z} d z
$$

In the formula, $\mu_{p e}$ is the photoelectric absorption coefficient of fluorescent photons in the scintillator film.

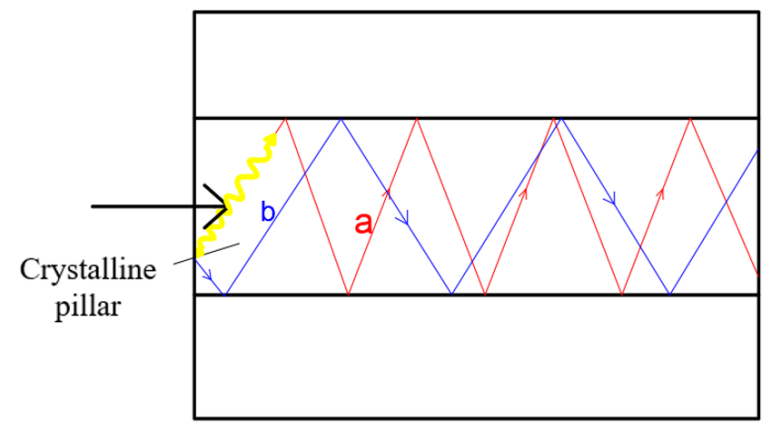

Figure 3 Schematic diagram of type A fluorescence propagation

In an ideal continuous layer of scintillator film, since fluorescent photons are emitted in all directions with equal probability, the probability of a certain fluorescent photon emission direction in the cone solid angle element $\theta \sim \theta+$ $d \theta$ can be calculated as:

$$
f_{\theta}(\theta) \mathrm{d} \theta=\frac{1}{2} \sin \theta d \theta(0 \leq \theta \leq \pi)
$$

Because the fluorescent photons are partially absorbed by the film during the transmission, the linear absorption coefficient of the film for photons during the process is $\sigma$, and the reflection coefficient of the reflective film $\mathrm{Al}$ is $R_{A l}$. Then the transfer function of the fluorescent photon with the exit angle $\theta$ generated by the X-ray excitation at the depth $\mathrm{z}$ can be expressed as:

$$
f_{p}(z, \theta)=\left\{\begin{array}{cc}
e^{\frac{L-z}{\cos \theta}} & \left(0 \leq \theta \leq \frac{\pi}{2}\right) \\
R_{A l} e^{\frac{L+z}{\cos \theta} \sigma} & \left(\frac{\pi}{2} \leq \theta \leq \pi\right)
\end{array}\right.
$$

According to the Monte Carlo method, the above ratio is integrated on $z$, and the probability and transfer function are integrated on the angle $\pi$ to obtain the mean value of the fluorescence transmittance $T_{p}\left(L, \sigma, R_{A l}\right)$, that is, the solution to the problem with Monte Carlo method. Here $L$ is the thickness of the film.

$$
T_{p}\left(L, \sigma, R_{A l}\right)=\int_{0}^{L} f_{z}(z) d z \int_{0}^{\pi} f_{\theta}(\theta) f_{p}(z, \theta) d \theta
$$


According to the formula of total reflection, the critical angle when the total reflection of the fluorescent photon occurs in the crystalline pillar is about $34^{\circ}$. Therefore, when the incident angle of fluorescent photons is larger than $124^{\circ}$ or less than $34^{\circ}$, it will all propagate in the crystalline pillar. As shown in Figure 3, both type A and type B light can be totally reflected in the crystalline pillar. Type B fluorescence is collectively referred to as type A fluorescence. For type A fluorescence, the angle between it and the crystalline pillar does not change when it spreads in the crystalline pillar, and the path length of the fluorescent photon is equal to the path length in the ideal continuous layer, so the transmitted rate of the fluorescence photon in the ideal continuous layer is still applicable in type A fluorescence.

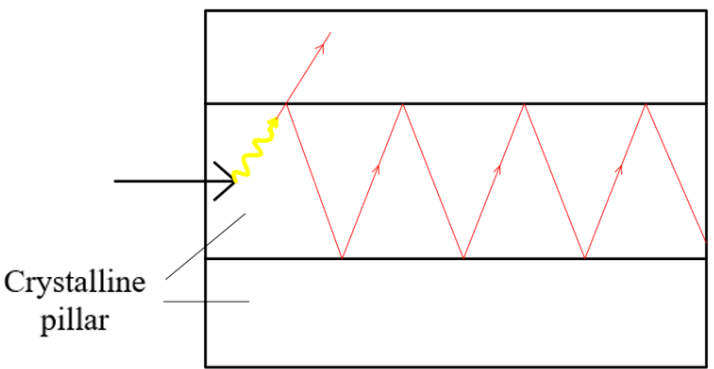

Figure 4 Schematic diagram of type B fluorescence propagation

When the emission angle of the fluorescent photon on the inner wall of the crystalline pillar cannot satisfy the total reflection, such as the fluorescence in Figure 4, this type of fluorescence should account for $17 \%$ of the total fluorescence. We refer to this type of fluorescence as type B fluorescence. Because this type of fluorescence cannot be totally reflected, B fluorescence will pass through the crystal pillars and propagate in the gap between the crystal pillars. Under ideal circumstances, we consider that the gap between the crystal pillars is extremely narrow. In this case, type B fluorescence can be regarded as a relay in a continuous ideal layer, and its light transmittance can still be expressed by formula (7).

In the actual process, there will be some semicrystalline pillars. When the fluorescent photons propagate in this kind of pillars, they will be reflected between the pillars and the reflective layer. We refer this type of fluorescence as type $\mathrm{C}$ fluorescence, as shown in Figure 5.

The transfer function of type $\mathrm{C}$ fluorescent photons is:

$$
f_{T C}(z, \theta)= \begin{cases}R_{c 1} e^{\frac{L-z}{\cos \theta} \sigma} & \left(0 \leq \theta \leq \frac{\pi}{2}-\theta_{L}\right) \\ R_{c 2} e^{\frac{L+z}{\cos \theta} \sigma} & \left(\frac{\pi}{2}+\theta_{L} \leq \theta \leq \pi\right)\end{cases}
$$

Among them, $R_{c 1}$ and $R_{c 2}$ are the reflection coefficients on the Al substrate and the Al wall, respectively, and $\theta_{L}$ is the critical angle of total reflection.

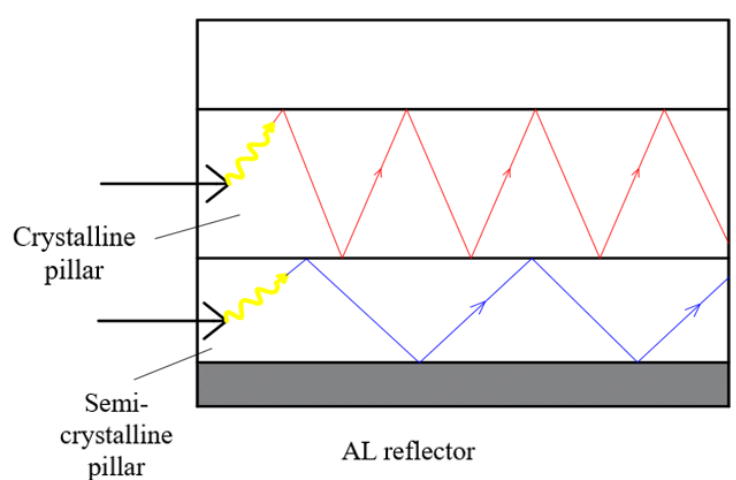

Figure 5 Schematic diagram of $\mathrm{C}$ type fluorescence propagation

\section{Simulation results and analysis}

\subsection{Simulation results}

The simulation results of the microcrystalline pillar structure are shown in Figure 6.

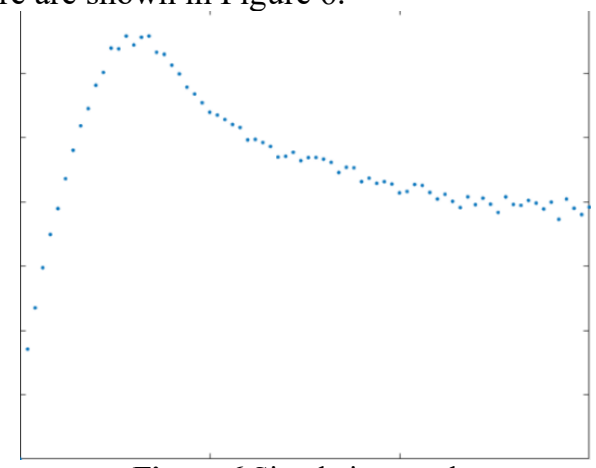

Figure 6 Simulation results

\subsection{Result analysis}

Results can be seen from the above figure as follows.

When the other parameters remain unchanged, the luminous efficiency gradually reaches the peak value with the increase of the film thickness, and then gradually decreases with the increase of the film thickness, and the rising speed is faster, and the falling speed is slower.

The reason why the initial luminous efficiency increases with the increase of the film thickness is that the luminous efficiency of the film is in a saturated state at this time, and a large number of $\mathrm{X}$ particles have almost excited all the photoelectric effects that can occur. In this case, as the film thickness increases, more X particles have a photoelectric effect, and the luminous efficiency increases rapidly. The reason for the subsequent decrease is that the film thickness continues to increase after reaching the peak, which will cause the length of the path of the excited fluorescent particles to travel in the film, increase the loss, and increase the number of absorbed particles, resulting in a slow decline in luminous efficiency. 


\section{References}

1. Structure and scintillation properties of $\mathrm{CsI}(\mathrm{Tl})$ epitaxial layers[J] . A Ananenko,A Fedorov,P Mateychenko,V Tarasov,Yu Vidaj. Applied Surface Science . 2004 (1)

2. The sensitivity and spatial resolution dependence on the microstructures of CsI:Tl scintillation layer for Xray imaging detectors $[\mathrm{J}]$. Bo Kyung Cha,Jun Hyung Bae,Chae-hun Lee,Sungho Chang,Gyuseong Cho. Nuclear Inst. and Methods in Physics Research, A. 2010

3. Structured CsI ( $\mathrm{Tl})$ scintillators for x-ray imaging applications. Nagarkar, V.V,Gupta, T.K,Miller, S.R,Klugerman, Y,Squillante, M.R,Entine, G. IEEE Transactions on Nuclear Science . 1998

4. X-ray and charged particle detection with CsI (Tl) layer coupled to a-Si:H photodiode layers. Fujieda, I,Cho, G,Drewery, J,Gee, T,Jing, T,Kaplan, S.N,Perez-Mendez, V,Wildermuth, D,Street, R.A. IEEE Transactions on Nuclear Science 1991

5. Dewetting behavior of CsI layers on LiF substrate[J] . A. Fedorov,A. Lebedinsky,P. Mateychenko. Journal of Crystal Growth . 2010 (1)

6. Studying the response of a plastic scintillator to gamma rays using the Geant4 Monte Carlo code[J] . Rasoul Ghadiri,Jamshid Khorsandi. Applied Radiation and Isotopes . 2015 